\title{
Spatial distribution of surface hoar crystals in sparse forests
}

\author{
C. Shea ${ }^{1}$ and B. Jamieson ${ }^{1,2}$ \\ ${ }^{1}$ Department of Geoscience, University of Calgary, 2500 University Drive NW Calgary, Alberta, T2N 1N4, Canada \\ ${ }^{2}$ Department of Civil Engineering, University of Calgary, 2500 University Drive NW Calgary, Alberta, T2N 1N4, Canada
}

Received: 21 May 2009 - Revised: 26 October 2009 - Accepted: 25 May 2010 - Published: 25 June 2010

\begin{abstract}
Surface hoar size and location relate directly to avalanche initiation trigger points, and they do so in smallscale spatial distributions. Physically, surface hoar will grow where the snow surface is cold relative to the air and water vapour is plentiful. Vapour aside, snow cools at night primarily by longwave radiation emittance. Emittance can be restricted by clouds, trees, and terrain features. With 96 independent spatial point samples of surface hoar size, we show the extreme small-scale size variation that trees can create, ranging from 0 to $14 \mathrm{~mm}$ in an area of $40^{2} \mathrm{~m}^{2}$. We relate this size variation to the effects of trees by using satellite photography to estimate the amount that trees impinge on sky view for each point. Though physically related to longwave escape, radiation balance can be as difficult to estimate as surface hoar size itself. Thus, we estimate point surface hoar size by expected maximum areal crystal size and dry terrain greyscale value only. We confirm this relation by using it at a different area and in a different formation cycle. There, its overall average error was $1.5 \mathrm{~mm}$ for an area with surface hoar sizes ranging from 0 to $7 \mathrm{~mm}$.
\end{abstract}

\section{Introduction}

Surface hoar crystals grow on the surface of snow from direct deposition of water vapor. The crystals, once formed and buried under additional snow load, form a persistent and brittle weak layer (McClung and Schaerer, 2006) with a distinct ability to propagate fractures within the snowpack and thus release avalanches.

As the surface hoar crystals collapse and slide in layer failure, the size of the crystals themselves may contribute to increased potential energy release and increased propagation of layer failure (Jamieson and Schweizer, 2000; Heierli et al., 2008). In addition, larger crystals maintain an unstable sepa-

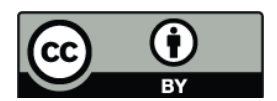

Correspondence to: C. Shea (cashea@ucalgary.ca) ration between slab and bed surface longer during the process of settlement and bonding (Jamieson and Schweizer, 2000).

In other words, an ability to predict where surface hoar crystals form and whether they are large or small would directly help predict and clarify where skiers may trigger slabs overlying a surface hoar layer. The scale of interest for such predictions lies at the skier scale, that is, at a small enough scale where we may say something useful about trigger points.

This paper focuses on the where rather than the when of surface hoar - often, avalanche forecasting operations have a good idea about whether or not surface hoar has formed, but desire additional information about the spatial distribution of the layer. Here, we examine the size of surface hoar crystals over small areas in sparse forests.

Besides being common skiing terrain in North America, trees provide control for many environment variables. Sparse forests offer protection from high winds, and they create extreme variation in net snow surface longwave radiation escape. Thus, such areas are known for being protected enough to grow surface hoar, but variable enough to not grow it homogeneously. Logging cuts serve as a prime example (McClung and Schaerer, 2006). In addition, understanding the variation of surface hoar in sparse forests has direct useful value: between 1984 and 1996, of the 13 fatal avalanches that released on buried surface hoar for which vegetative area is known, six of them happened at treeline or below (Jamieson and Geldsetzer, 1996).

Physically, we expect that the size of surface hoar can be directly and spatially correlated with sky view, a measure of how much atmospheric view and exposure a point on the snow surface has, and through which it may emit longwave radiation. In forests, close trees over a point on the ground decrease sky view, whereas widely spaced trees increase it. As sky view is difficult to measure point-by-point over large areas (Brown et al., 2001), we approximate it by using greyscale values in dry land satellite photography where darker pixels correspond to trees. 
We begin with an overview of previous studies of physical parameters in surface hoar formation. Our methods, including use of land imagery, follow. Then, we present options for analysis: as this study is the first to attempt spatial surface hoar size prediction without accompanying weather data, we show the avenues that led us to use satellite photography. Finally, we present our empirical model, its validation, and a summary and outlook.

\section{Previous work}

Whereas previous studies have linked surface hoar growth to bulk transfer of water vapour, few have examined net longwave radiation effects and none have done so in a twodimensional spatial setting at the skier scale.

Many factors affect surface hoar growth, from wind (Hachikubo and Akitaya, 1997; Hachikubo, 2001; Föhn, 2001), to radiation balance (Cooperstein, 2008), to air-snow temperature gradients (Lang et al., 1984; Hachikubo and Akitaya, 1997), to humidity (Feick et al., 2007). At the most simple physics level, one might say surface hoar grows when we have cold ice, warm air, and lots of water vapour. However, some factors affect growth both positively and negatively. Clouds, for example, supply vapour but reduce effective longwave radiation escape (Colbeck et al., 2008).

Surface hoar needs a substantial amount of water vapour. This explains the success of bulk transfer models (Colbeck, 1988; Hachikubo and Akitaya, 1997; Höller, 1998; Föhn, 2001; Lehning et al., 2002): wind can provide moisture to the snow surface via bulk transfer. However, humidity does not always serve as the main determining factor. Previous work showed similar air temperatures and relative humidity both within and out of forests (Höller, 1998), but we observe high surface hoar size variance within forests. So, if air temperature and vapour presence do not change spatially within sparse forests, the remaining varying factors should be wind - or bulk transfer capacity - and longwave radiation escape.

Wind presents its own particular set of challenges. Some studies found light wind to be beneficial to surface hoar growth (Föhn, 2001; Hachikubo and Akitaya, 1997; Colbeck, 1988). Yet, there are recorded examples of wind (with relative humidity close to that of vapour pressure over ice) sublimating ice rather than depositing vapour upon it (Hachikubo, 2001; Feick et al., 2007). In addition, the physical necessity of wind in the deposition process itself remains unresolved, with conflicting opinions in Lang (1985) compared with Hachikubo and Akitaya (1997) and Föhn (2001). At the very least, high $\left(>3 \mathrm{~m} \mathrm{~s}^{-1}\right)$ wind speeds at the snow surface will affect the shape of deposition into something other than surface hoar (Cheng and Shiu, 2002), and low wind speeds will present large barriers to bulk transfer of adequate vapour.

Wind affects both major factors in surface hoar growth temperatures and vapour - and it does so in a currently unmodellable spatial fashion at useful scales (Hägeli and Mc-
Clung, 2000; Campbell et al., 2004). For this reason, most of the studies mentioned above involve measurement points only very near weather stations or other monitoring equipment such as radiometers. Here, then, we have come to an apparent impasse: our best mechanisms for estimating surface hoar size require accurate wind and humidity measurements, and neither may be predictively mapped, spatially, within a $40^{2} \mathrm{~m}^{2}$ area. Thus, for this first spatial predictive effort, we chose an environment that controls the variance of both.

This leaves the option for a spatial study on the variance in effective longwave radiation escape. Longwave radiation effects have already been studied in relation to surface hoar growth by aspect (Cooperstein, 2008) and integrated with bulk transfer-based estimates for better predictions in open areas (Lehning et al., 2002).

An excellent location to study these longwave effects is in sparse forests. Trees block sky view for a point on the ground. Trees are detectable - individually - over large areas using modern basic satellite photography. Trees are usually stationary over long time scales, have high emissivity (which makes them quite good at re-radiating longwave escape from snow), and vary spatially on scales that humans can access, measure, and visualize. Furthermore, trees offer the conditions desired here - that is, protection from high winds.

Finding a clarifying situation, such as sparse forests, with which to study sky view and longwave blockage effects can be quite helpful. The self-compounding nature of physical factors involved in surface hoar growth - as well as their small-scale variability at any give point - have put up great obstacles to studying surface hoar growth by point sampling sparsely over a large area (Feick et al., 2007). Also because of this, very few studies have tried to map the variance of these effects over terrain, and none - until now - have attempted spatial prediction.

\section{Methods and data}

This paper contains two types of field measurements: surface hoar crystal sizes and snow surface point temperatures. We also compare these field data to satellite images to obtain a measure of closeness to trees. The methods for both obtaining the data and correlating the data and images are presented here. In our methods, we sought to:

- Provide a reasonable estimate of surface hoar crystal size variation over terrain at the skier scale.

- Utilize only information easily available to the practitioner: i.e., single point samples of surface hoar size and basic dry land satellite photography.

In other words, we intentionally present a method to estimate spatial distribution which uses limited input, few necessary resources, and no field instrumentation. 


\subsection{Field method and data}

In our area of operation in the Columbia Mountains, Canada, we point-sampled surface hoar sizes during the surface hoar formation periods between 16 January to 22 January, and 17 February to 20 February, 2009.

We spatially distributed the point samples using the Star sampling method (Shea and Jamieson, 2010). This ensured randomized spacing between points and snow surface preservation between sampling days. The random placement of points prevented the operator's preference from being influenced by terrain and other attributes of the area. In addition, the method allowed random spacing between analysis points; such randomness generally improves the robustness of spatial analyses later done with that data (Kronholm and Birkeland, 2007). The slope preservation enabled by Star's layout allowed us to measure the change in surface hoar size at the same locations from day to day.

Sample point distance separation ranged randomly from $10 \mathrm{~cm}$ to the full width of a sample area. Each sample area contained 48 crystal size samples and covered approximately $40 \times 40 \mathrm{~m}$ of terrain. Due to the fractal nature of snow (Deems et al., 2006), we chose our extent carefully to be one that could capture the scale of skier triggering.

At each point, we recorded the minimum and maximum size of surface hoar crystal found there, which we later averaged into a mean size for that point. We noted a size of $0 \mathrm{~mm}$ only if no crystals were found. The same points were sampled repeatedly on consecutive days during the January cycle.

The efficiency of the sampling design allowed us to obtain more spatial points than any previous surface hoar study. In short, 96 spatially separate and semi-random points were obtained in the January cycle, and each point was visited three times over four days. In the February cycle, 48 spatially separate points were obtained, and they were each visited once.

The two sample areas in January, from which we developed the relation with vegetative greyscale in terrain imagery, need be distinguished. We will call them Area I (primarily north facing) and Area II (primarily northeast facing). Both lie at an elevation of $1900 \mathrm{~m}$, with generally horizontal orientation but containing small slopes up to 25 degrees of incline. Neither sample area had dense forest, and neither was entirely open, although Area II had more open area than Area I. One can see a photo of the general terrain in Fig. 1. Each contained a mix of trees and rolling terrain, which meant any immediate obstructions to sky view were of a smaller size than the sample area.

The one sample area in February, which we used to confirm the relation with greyscale, is located at a different location $(150 \mathrm{~km}$ to the northwest), and a different aspect (south facing). The attributes of the area were similar to Areas I and II in all but aspect, that is, a mix of trees and small terrain features.

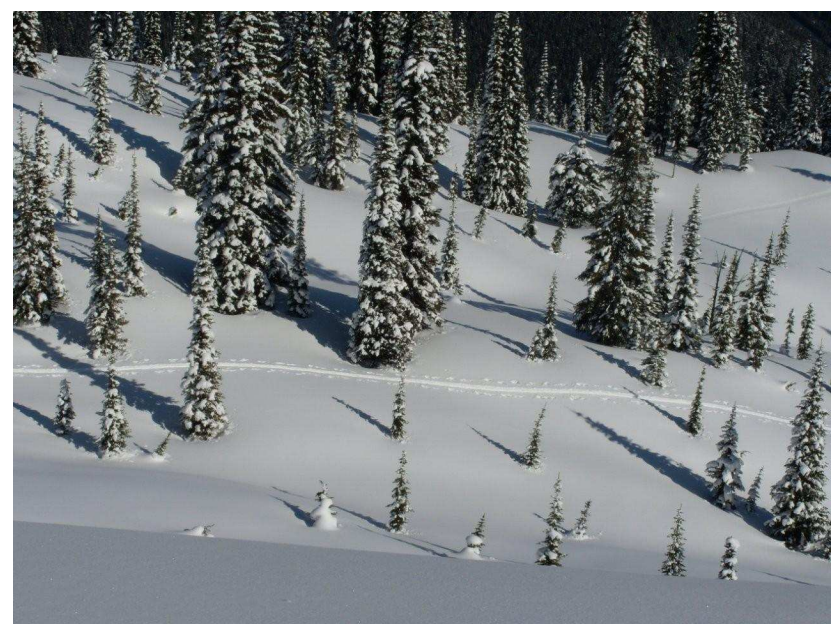

Fig. 1. Photo of a portion of Area I. Notice the ski tracks across the area for scale. This area is quite sheltered from wind. Also, during the January surface hoar cycle, the winds in the area ranged from only 1 to $12 \mathrm{~km} \mathrm{~h}^{-1}$.

\subsection{Temperatures}

For our temperature measurements, we measured the surface temperature of the snow at each point with two different infrared thermometers - a Thermohawk 400 hemispherical 1:1 thermometer used at $1 \mathrm{~m}$ above the surface, and a Testo 825-T4 3:1 thermometer used at $30 \mathrm{~cm}$ above the surface. This occurred only for the last January cycle day, for both Areas I and II. As both measurements had similar results, we present the downward facing measurements from $30 \mathrm{~cm}$ above the snow surface. What we call point snow surface temperatures, then, are effectively $10-\mathrm{cm}$ diameter averaged snow surface temperatures.

\subsection{Imagery}

Satellite photos of Areas I and II appear in Fig. 2b and d, with the blurry dark areas corresponding to trees. We obtained our land cover imagery from Google Earth. Individual trees can be distinguished; for freely available data, these photographs are quite good. The images exhibit no snow on the ground or trees; we use them only for greyscale values to estimate sky view. The trees are primarily conifers, which can provide dependable greyscale shading distinctness.

From a screenshot of the satellite image overlain on Google Earth terrain, we extracted the terrain-projected (warped) image area corresponding to each of the two sampled areas. We then used cubic interpolation to scale the projected areas back into flat rectangular form. No other image modifications besides a projection to a flat rectangular surface occurred. Area II required more interpolation than Area I to make it rectangular, with approximately 25 degrees of stretch in the lower right hand corner, and smaller adjustments in the other corners. 

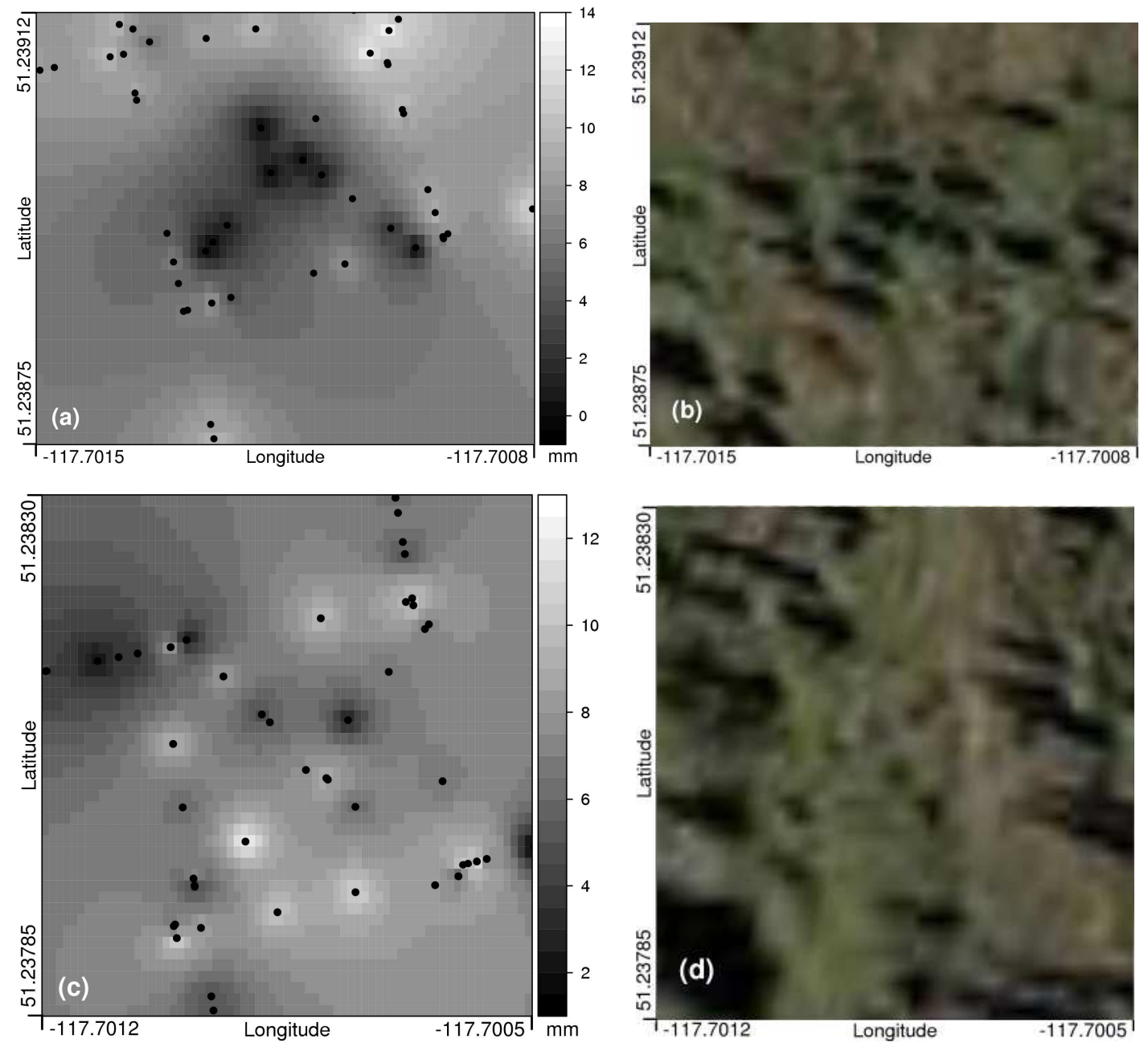

Fig. 2. Visual overview of the two sample areas. (a) IDW map of mean crystal sizes for one day at the Area I location, (b) satellite image corresponding to Area I. (c) IDW map of mean crystal sizes for one day at the Area II location, (d) satellite image corresponding to Area II. For (a) and (c), black dots indicate the actual physical sample locations. For (b) and (d), images are provided under the Google Earth terms of use, copyright 2009 TeleAtlas, 2009 British Columbia, and 2007 Google Earth.

Each Star sample area of approximately $40 \mathrm{~m} \times 40 \mathrm{~m}$ translated to a satellite image with about 10 pixels per $1 \mathrm{~m}$ resolution. However, this includes pixels generated by cubic interpolation when re-projecting the image to a rectangular form.

Greyscale shading in our photographs comes primarily from trees, less so from rocks and terrain rolls, and nearly nothing from steep slopes or aspect within a given small area. This gives a clean, qualitative link to sky view: areas of lighter grey in a small area generally have more, and areas of darker grey within a small area generally have less.

\subsection{Mapping}

To make visual maps of the crystal size and surface temperature measurements shown in this paper, we created a continuous image of probable values from our real-world point measurements. This step is simply to make it easier to visually comprehend the measurements; all data points involved in calculations in this paper are only those actually physically measured in the field.

To produce these visual maps, we used the standard inverse distance weighting (IDW) algorithm. IDW weights the values of closer known points more heavily than further known points, where the weight $w$ of each known points with distance $d$ from the point we wish to predict gets included in the average with a weight $w(d)=d^{-2}$. We used IDW to calculate size and temperature values at all locations on a $1 \times 10^{-5}$ spaced latitude and longitude WGS84 grid (Pebesma and Wesseling, 1998). Two such example maps can be seen in Fig. 2a and c. 


\subsection{Determining position}

We determined the latitude and longitude of the sampled point locations using the following information:

- Between 600 and 1400 GPS reference points per visit to each sample area.

- Pacing each transect of sample points (Star consists of six transects) using pace length and various measuring devices.

- Recording reference information in addition to crystal size at each point, including patchiness of surface hoar crystals at each point, which was used for correct dayto-day ordering of points.

These independent sets of information essentially served as checks to assess the accuracy we could obtain from any one of the measurement methods. Our base fitting method consisted of a custom-designed GIS fit program, which determined the position of each sample location by closest fit from the average of thousands of compiled track points.

We estimate that our total cumulative error per 8-point transect line with GPS and pacing error combined (and practically inseparable) ranged between $1 \mathrm{~m}$ and $5 \mathrm{~m}$ depending on the sample. We used a relatively accurate recreational handheld GPS unit to let us not only move quickly while sampling, but also to demonstrate that such a device can provide position for the field measurements needed for others to use the method outlined in this paper.

When determining position on the satellite photography, we moved the coordinates of Area II, as a unit, seven meters to the south. This occurred due to visual match up of tree location values recorded in the field with what we observed on the Google Earth imagery. This shift was most probably needed because of the different tilt of the trees in the satellite photograph, creating longer shadows in the image. Area I required no translation, and we did not perform any translation on the sample from 19 February 2009 to let it serve as an evaluative dataset.

\section{Options for analysis}

Where weather data is concerned, many-point spatial studies face a choice of challenge. They may use measurement equipment at every point, which can be expensive and time consuming. Or, they may model and extrapolate values from nearby measurement equipment, which can result in loss of accuracy.

With our intent to develop a model which uses readily available inputs, we discovered that, in our data, crystal size generally scales with satellite photography greyscale. This is not to say that greyscale scaling will always work. Rather, we show here that it worked in our conditions, which we consider to be typical of sparse forests. Furthermore, the use of greyscale shows that other, non-weather data may be used to creatively augment, improve, or even provide estimates. We did not arrive at such a solution immediately, and the following subsections outline our process.

\subsection{Variograms}

As an exploratory method for discovering spatial structure and process scales, the variogram (Cressie, 1993) currently has no peer. Conservatively, one may determine the process scales for a given process and area, and then return to that area and scale a sampling method to capture that process on its scale of operation as accurately as possible. Oftentimes, however, one does not have that luxury as snow sampling can be destructive and the desired conditions last only a short time.

We could not re-scale our sample without further destruction to the slope. However, as we could return from day to day, we could find variograms both for one day and for change across multiple days. Searches for both isotropic and anisotropic spatial correlation in the single-day surface hoar size sets did not reveal any obvious patterns. However, the multi-day variograms had more defined ranges, as these distill the data down to only the processes which change crystal size after formation. Even these variogram ranges were nondefinite. Figure 3 shows two such variograms.

The variable and weak nature of these variograms revealed two issues with the analytical use of the variogram here. First, the process scale does not necessarily stay constant from day to day, or area to area. As we can see in Fig. 3, even the weak ranges vary for the same analysis from day to day. One may intuit that trying to chase a constantly varying set of process scales would be quite time consuming, and would also be quite spatially consuming when the sampling involved is a destructive process.

Second, the type of sampling method and the scaling of that sampling method greatly affect the outcome of a variogram. Due to the fractal nature of some snow measurements (Deems et al., 2006), a sampling method with points that oscillate with the process scale can greatly affect the outcome, and even pick up and mix up ranges of unexpected interrelated processes.

We cannot know whether either of the above issues caused the lack of good variogram results. However, our sampling method evaluation (Shea and Jamieson, 2010) and close measurement point spacing give weight to the variogram not being useful in these conditions. Furthermore, a variogram cannot help one spatially predict any value, unless the process creating the range can be very precisely mapped over the desired area.

\subsection{Surface temperature}

We also assessed the physical processes. We measured surface temperatures, since surface hoar grows on ice when it is cold relative to the air (Lang et al., 1984; Hachikubo 

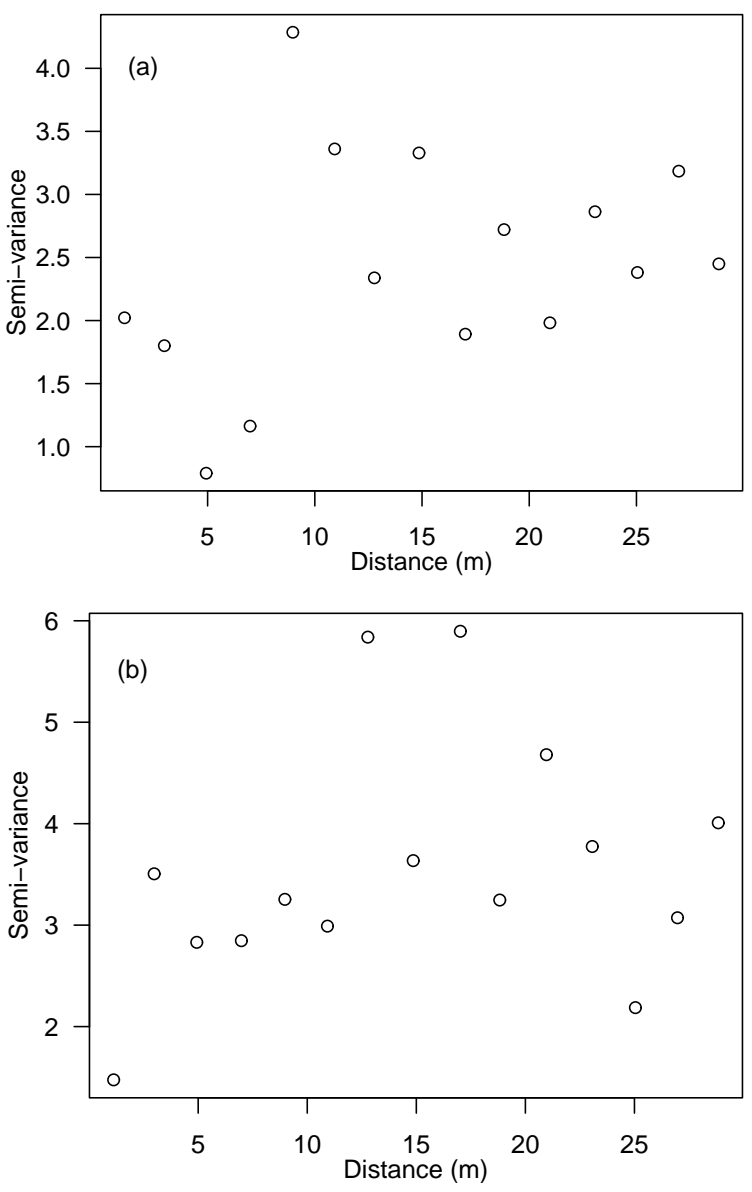

Fig. 3. Two examples of size-difference semi-variograms with weakly defined ranges. (a) Overnight size change from 16 to $17 \mathrm{Ja}-$ nuary, Area II. (b) Overnight size change from 17 to 18 January, Area I.

and Akitaya, 1997). At the end of the January formation cycle, we took a nighttime point surface temperature (04:0006:00 local time, LT) and daytime point surface temperature (10:00-12:00 LT) at every one of the 96 spatial points in Areas I and II. We show crossplots of surface temperature versus crystal size in Fig. 4.

The correlation between nighttime surface temperature and crystal size ranged from $r=-0.64$ to -0.74 , both $p<0.001$. The correlation between daytime surface temperature and crystal size ranged from $r=-0.39(p=0.03)$ to $r=-0.31(p=0.06)$. This supports the findings of Cooperstein (2008): a single temporal measurement of the effects of daytime shortwave matters less than the overall sum of shortwave, which a night of longwave escape must then overcome. However, this result does not necessarily help with size prediction, for the following reasons.

First, the process of directly predicting such point temperatures at small scales over terrain would be very complicated. Though current work has begun to tackle this very problem
(Morstad et al., 2007), much work remains. The magnitude of those efforts should demonstrate the complexity of building and verifying a usable physical predictive model for temperatures alone, much less for surface hoar size.

One also may think to use trees via greyscale to predict the more physical variable of surface temperature, and then use the temperature predictions to estimate size. However, the relationship between distance from trees and point surface temperature do not necessarily correlate. For example, during the day, open and non-treed snow absorbs more incoming shortwave radiation, and thus must emit all of that radiation and more at night to achieve the same surface temperature. Snow close to trees, on the other hand, gains some protection from incoming shortwave during the day. From only these effects, larger surface hoar would grow within trees, which we do not observe in practice.

The more dominant effect of trees on nearby snow is probably a reduced ability for the snow to effectively vent heat via longwave radiation escape at night due to tree blockage and re-radiance. Trees also can create canopies which keep the air warm by buffering it from temperature changes (Sicart et al., 2004), an important factor in near-surface temperature gradients but not accounted for by surface temperatures alone.

Even if we were to be able to estimate point surface temperatures at small scales over terrain, the strong size correlation solely with nighttime temperatures indicates that a radiation balance estimate would need to not only be accurate in space, but also quite accurate in time, compounding the difficulty. One may examine the spatial IDW maps of the change in surface temperatures from night to day in Fig. 5. Even during the daytime, some areas cooled and some areas warmed in a complex interplay of shadows and radiation balance. The most noticeable surface warming actually occurred at shaded points within the trees, as one may see by comparing the temperature plots in Fig. 5 with their corresponding images in Fig. 2.

Furthermore, this sets aside the issue that surface temperatures account for only part of the variance in size, as most natural variables do. Estimating temporal surface temperatures at such small scales with all of the complexity mentioned above presents a great deal of difficulty for the predictive benefit of only one variable. So, we sought an empirical approach.

\subsection{Greyscale}

Trees affect skyview - which in turn influences diurnal surface temperature changes - and trees also affect air temperatures, wind, and so on. They capture the effects of many variables in one physical construct. Due to surface hoar needing efficient longwave radiation cooling, we expect that, all other things being equal, surface hoar will be smaller near trees and larger in open clearings. 

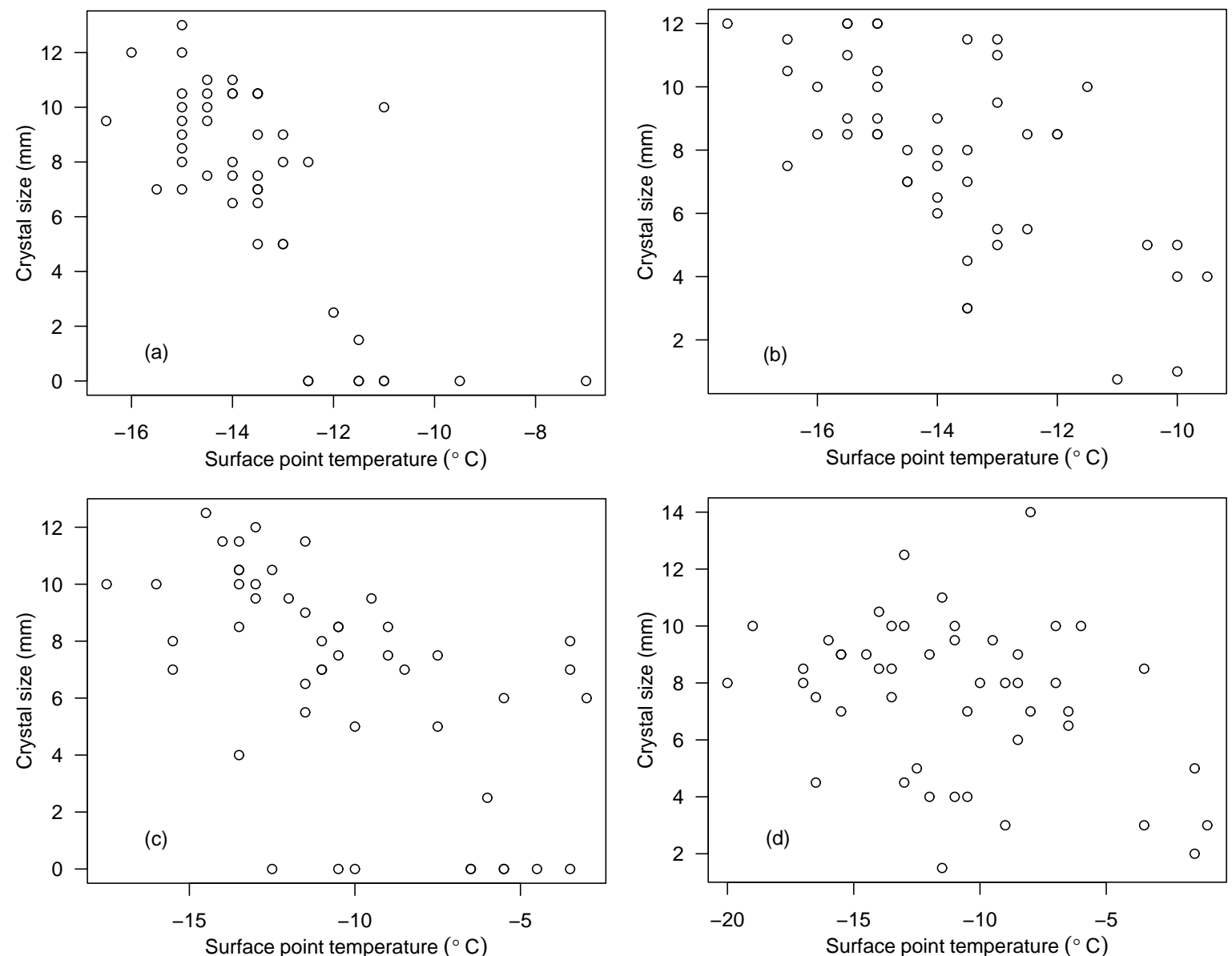

Fig. 4. Cross plots of point snow surface temperatures versus mean surface hoar crystal size found at each point during both night and day. (a) Night, Area I. Pearson correlation $r=-0.74, p<0.001$. (b) Night, Area II. Pearson correlation $r=-0.64, p<0.001$. (c) Day, Area I. Pearson correlation $r=-0.39, p=0.03$. (d) Day, Area II. Pearson correlation $r=-0.31, p=0.06$.
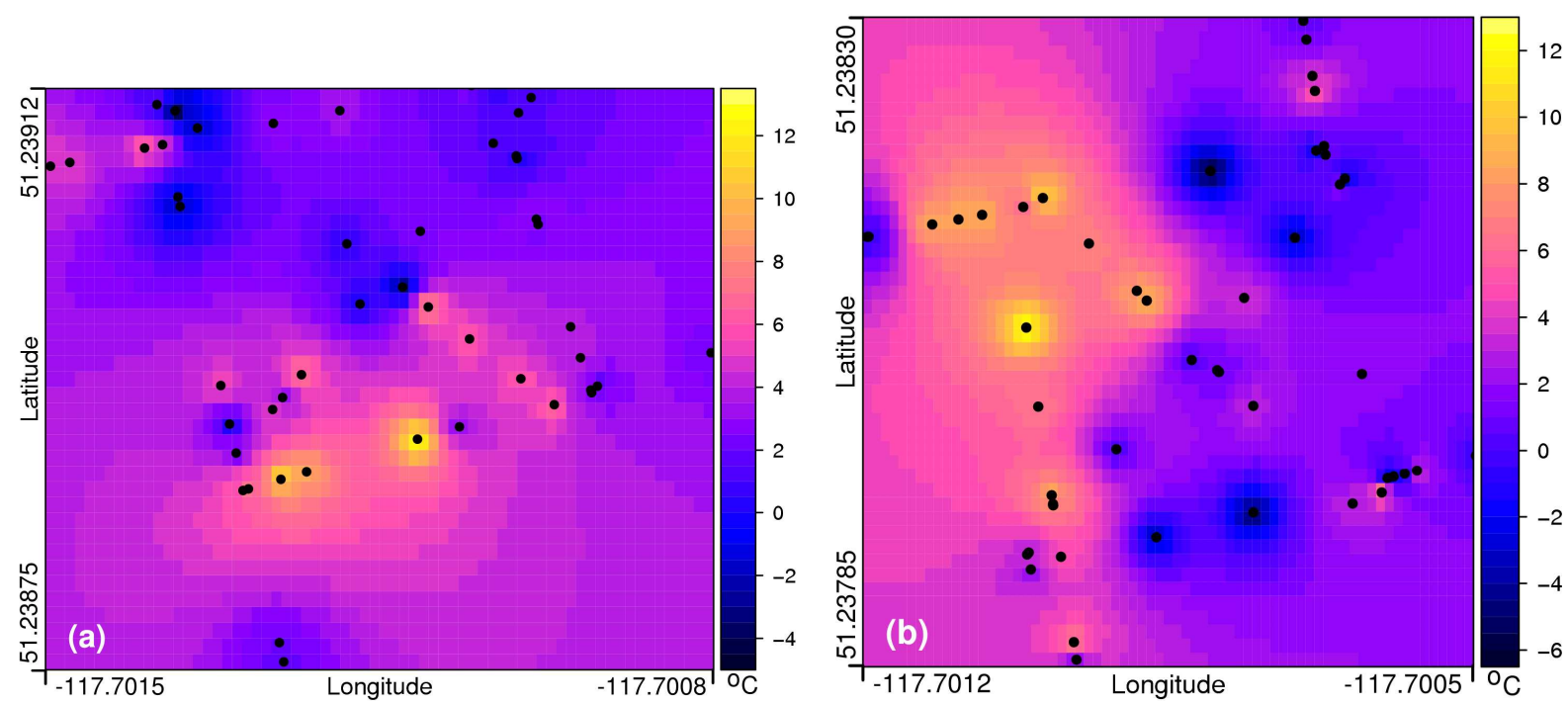

Fig. 5. Inverse-distance weighted plot of temperature change from night (approximately 05:00 LT) to day (approximately 11:00 LT) on 21 January 2009. Black dots indicate the locations of the actual sample points. (a) Area I. (b) Area II. 

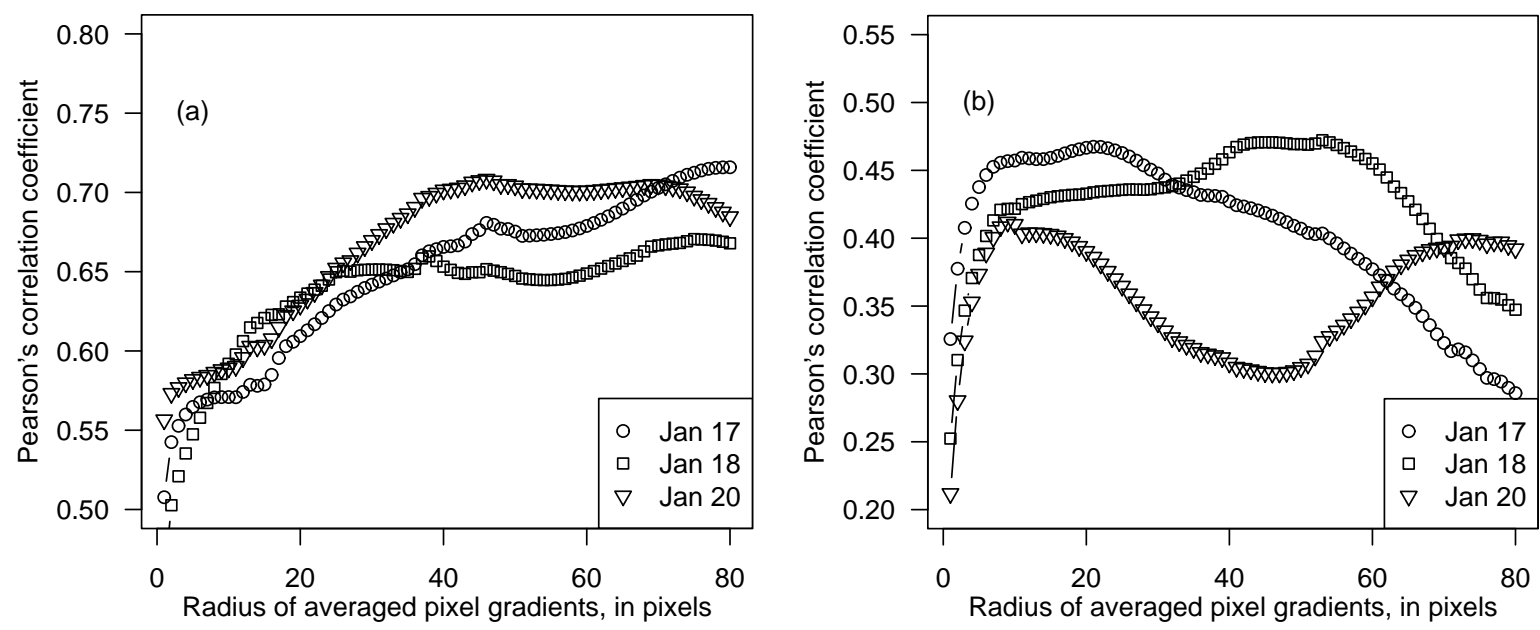

Fig. 6. Pearson's correlation coefficient variation by radius of satellite image pixels included in a point averaged greyscale value. Each correlation coefficient shown comes from the 48 points per given day. The values for grey 40 correlation lie at $x=41$. (a) Area I. All correlations have significance $p<0.05$. (b) Area II. All correlations except the three points furthest left on each line have significance $p<0.05$.

To verify this empirical relation, we used the greyscale values of dry land satellite photography. The point crystal size measurements and corresponding satellite imagery for the two January formation cycle areas are shown in Fig. 2. We expect that a single pixel on the satellite photo, due to noise and extreme sensitivity to local effects, will have poor correlation to a single crystal point measurement. Rather, for purposes of both reducing the effects of noise and accounting for a finite area around a geographical point, we averaged a given radius of greyscale pixels.

To find the best such radius, we constructed eighty different greyscale point sets, each containing one pixel value per measurement point, and each corresponding to a different radius - one to eighty - in linearly averaged greyscale pixels. For a radius of 10 pixels, for example, we averaged the block of 21 by 21 pixels surrounding the sample point to produce our averaged point greyscale value. This occurred independently of nearby or overlapping sample radii. We then compared the correlation between the mean surface hoar size at each measurement point and the averaged greyscale values within a given radius in the satellite image at that point. The fluctuations in correlation values by radius can be seen in Fig. 6.

Qualitatively, one may think of this as the area of effect that trees might have on a surface hoar crystal or, inversely, as an empirical measure of sky view. In Fig. 6, one can see that an obvious maximum exists on some days but not always, and the maximums do not always occur at the same radius value.

Our choice of radius was the maximization of correlation over the six days, which lies at approximately 40 pixels. Though this general maximum is obviously not individually true for 20 January 2009, 40 pixels gives us an easy to use and easy to generate value which has reasonable correlation. Compared to the effort of predicting spatial surface temperatures, for example, this radius-of-tree-effect variable gives straightforward values. The 40 pixel radius corresponds to an 81 pixel diameter, or an area with a radius of about four meters on the imagery used here. We call this averaged greyscale value over a 40-pixel radius grey $_{40}$.

The potential drawbacks to this method are also readily apparent. In open areas, where this qualitative relation between skyview and greyscale variation does not hold, this radius of effect also would not hold. However, for the purpose of sparse forests, it cleanly isolates the amount of tree cover around a point and has good correlation to surface hoar size.

\subsection{Regression}

Having found an easily and spatially obtainable value grey $_{40}$ - we wish to use it to do something spatially useful. We first constructed six different linear models using basic linear regression with unit least squares and utilizing $\mathrm{R}(\mathrm{R}$ Development Core Team, 2006). These six models correspond to using grey 40 as the independent variable on each of the three sample days and two areas. We captured the slope $a$ and intercept $b$ of each linear regression model.

The impracticality of using an ideal day as a model for other days became readily apparent. The slope and intercepts of each of the six models varied at least partially because the average maximum surface hoar size for each sample area varied (14.7 mm for Area I, and $12.5 \mathrm{~mm}$ for Area II). Determining which of these maximum sizes represented the ideal could not be easily done. In addition, some days appeared to have a more curved (exponential) relationship with greyscale, whereas the overall relationship with greyscale was linear. 
Further, the range of grey 40 varied $(0.177$ for Area I, 0.156 for Area II), as did the other averaged grey point values. Even when slopes and intercepts were scaled by maximum crystal size and grey 40 , the slopes of greyscale luminosity versus crystal size varied from 26.23 to 69.49 . Regression over the whole 288 point dataset and individual areas fared somewhat better, with the standard deviations of the residuals just over $3 \mathrm{~mm}$.

Regression depends very strongly upon each individual smaller dataset being affected by the independent variable in the same numerical way. For example, if trees affect crystal size very strongly one day and less strongly the next, regression across the two datasets would average the effect and potentially produce an non-useful result. Conversely, regression can overfit to the effect of a single day and area. We see that here: even with a generally good relationship to the independent variable ( grey $_{40}$ ), both slopes and normalized slopes were so overfit to a particular day as to not be useful.

Trees stay constant from day to day, and so their type of effect on snow should also stay more or less constant from day to day. Exceptions exist: when the entire area is covered by a cloud, for example, trees would not be the dominating factor. But for the clear conditions which surface hoar benefits from (and which existed in this study) we expect trees to have the same general effect from day to day, area to area. So we turned toward developing a relationship for a given size increase per change in grey 40 .

\subsection{Constant increase}

Efforts with regression implied that we needed a way to scale, by area, for the range in both crystal size and greyscale. Both of those values do not stay constant across large areas, but as here we focus on small areas and even smaller scales of size estimation, they functionally serve our purpose. To capture the observed and generally linear relationship with grey ${ }_{40}$, we split the greyscale range for each area by the observed range in crystal size, e.g. range(grey 40$) /$ range(mean_sizes). This gives an amount of greyscale brightness increase per unit crystal size increase.

We applied this to the two areas sampled in January 2009. To do so, we needed a minimum mean crystal size $(0 \mathrm{~mm})$ and corresponding grey 40 value, as well as a maximum mean crystal size (14.7 mm for Area I or $12.5 \mathrm{~mm}$ for Area II) and a greyscale range (as stated above, 0.177 for Area I, 0.156 for Area II). With these values, we can determine a slope for a linear model to predict crystal size for each area. This type of scaling, though simple, adapts the expected crystal sizes to (a) the greyscale range of each area, and (b) the crystal size range in the area.

Both the January sample areas each had the same averaged minimum grey $_{40}$ at $0 \mathrm{~mm}$ size locations. This dark greyscale value was greybaseline $=0.22$. When all of this data was applied numerically to the January areas, it resulted in a surpris- ingly cross-day, cross-sample result of $\Delta$ grey $_{40}=0.012 / \mathrm{mm}$, or a $1.2 \%$ increase in greyscale whiteness for every additional $\mathrm{mm}$ of crystal size.

This constant increase strategy implies that a spatial size prediction within the area depends on:

- The dark, usually $0 \mathrm{~mm}$ crystal-producing grey 40 value called grey baseline. $0 \mathrm{~mm}$ does not necessarily have to serve as the minimum, but did for both surface hoar formation periods in this paper.

- The value of grey $_{40}$ - the 40 pixel radius greyscale average - on the image at the point we wish to predict, i.e. grey $_{40}($ lat, lon).

- $\Delta$ grey $_{40}$, which is the change in grey $_{40}$ value per expected $\mathrm{mm}$ of surface hoar size for the specific surface hoar layer and area. This part requires at least one (and, for these areas, only one) field measurement.

\subsection{Model}

A generalization of the constant increase relation gives our size prediction size predict $_{\text {at location (lat, lon) within the area: }}$

size $_{\text {predict }}($ lat, lon $)=\frac{\left(\operatorname{grey}_{40}(\text { lat }, \text { lon })-\text { grey }_{\text {baseline }}\right)}{\Delta \text { grey }_{40}}$

Using the above model means that we may estimate the mean size of a surface hoar crystal at the coordinates (lat, lon) by using only satellite image data and two empirical scaling numbers for the area: grey baseline and $\Delta$ grey $_{40}$.

For the case of Areas I and II during the 16-22 January 2009 surface hoar cycle, Eq. (1) can be rewritten as:

$\operatorname{size}_{\text {jan_16-22 }}($ lat, lon $)=\frac{\left(\text { grey }_{40}(\text { lat }, \text { lon })-0.22\right)}{0.012}$

To develop such a specific equation for an area, one primarily needs to find $\Delta$ grey $_{40}$ for that area. Since it equals the amount of positive change in grey 40 per expected $\mathrm{mm}$ growth of surface hoar, we need to obtain bounds on both the size and greyscale values for an area. Greyscale grey $40 \mathrm{~min}-$ imum and maximum bounds for the area are greybaseline and grey $_{\max }$, respectively. Surface hoar crystal size minimum and maximum values for the area are $\operatorname{size}_{\min }$ and size $\mathrm{max}_{\max }$, respectively.

With these values, one may obtain the $\Delta$ grey $_{40}$ for an area:

$\Delta$ grey $_{40}=\frac{\text { grey }_{\max }-\text { grey }_{\text {baseline }}}{\text { size }_{\max }-\text { size }_{\min }}$

Physically finding these values is likewise intuitable. For the greyscale values, greybaseline and grey ${ }_{\max }$ may be generally found via a histogram of grey 40 for an area. More accurately, they may be found in the image at the corresponding locations of the size $\mathrm{min}_{\min }$ and size max $_{\max }$ field samples. 
As for crystal sizes, field sampling may be done in the brightest grey $_{40}$ area of a $40^{2} \mathrm{~m}^{2}$ area to obtain size max $_{\max }$, and in a grey baseline dark area to obtain size $_{\min }$. Even more generally, two samples of size and greyscale may probably be obtained from any two locations varying in size and grey 40 values; however, using the expected minimum and maximum values for an area allows the capture of the widest range and thus a potentially more accurate coverage of that range.

Our experience indicates that the January surface hoar formation cycle was typical for the general area, and it allowed a simplifying step. For all days and both areas, the minimum surface hoar crystal size size min $_{\text {wa }} 0 \mathrm{~mm}$. This can be confirmed both visually in the images in Fig. 2, and by noting that, physically, trees emit longwave radiation and can block sky view enough to cause such $0 \mathrm{~mm}$ values.

This gives a more specific version of $\Delta$ grey $_{40}$ which we use in this paper:

$\Delta$ grey $_{40}=\frac{\text { grey }_{\max }-\text { grey }_{\text {baseline }}}{\text { size }_{\max }}$

Finally, when Eq. (4) is combined with Eq. (1), we obtain a more intuitive relation to the size obtained from an open area field sample:

size $_{\text {predict }}($ lat, lon $)=\operatorname{size}_{\max } \frac{\text { grey }_{40}(\text { lat }, \text { lon })-\text { grey }_{\text {baseline }}}{\text { grey }_{\max }-\text { grey }_{\text {baseline }}}$

As demonstrated by this relation, with this surface hoar formation pattern one needs minimal local knowledge to obtain the point sample of minimum or maximum mean size for a given surface hoar cycle. They may be found in most grey and least grey areas of a satellite image, respectively. Of course, one may already see instances where large scale processes would interfere with large scaling of this model. But as assessed in the next section, for our $40^{2} \mathrm{~m}^{2}$ sample areas with trees as the dominant factor, this concept held.

\section{Results}

We found that the model in Eq. (2), when used to predict dayto-day surface hoar sizes for the 16-22 January surface hoar cycle, showed reasonable results. The standard deviation of error for the January size predictions are less than half of the mean crystal sizes, generally giving $2.5 \mathrm{~mm}$ average error on $7 \mathrm{~mm}$ average crystals. A more intuitive interpretation would be that if one were to think of categories of crystal size, e.g. bigger or smaller, the greyscale results usually predicted the same category for a given point. Still, the model performed better than the regression approach described above.

Statistical summaries about the residuals between predicted mean sizes and actual mean sizes for all days in the cycle can be seen in Table 1. The higher mean size with lower maximum size in Area II reveals its more generally open terrain than Area I. In other words, crystals grow larger
Table 1. Comparison of residuals for both sample areas. Residuals are the difference (Actual Point Mean Crystal Size) - (Predicted Point Mean Crystal Size). Each sample area contains 144 actual point versus predicted point comparisons. Mean and maximum size values are from all observed sample points for each area.

\begin{tabular}{lcccc}
\hline & $\begin{array}{c}\text { Residual } \\
\text { mean } \\
(\mathrm{mm})\end{array}$ & $\begin{array}{c}\text { Residual } \\
\text { std dev } \\
(\mathrm{mm})\end{array}$ & $\begin{array}{c}\text { Mean } \\
\text { crystal size } \\
(\mathrm{mm})\end{array}$ & $\begin{array}{c}\text { Maximum } \\
\text { crystal size } \\
(\mathrm{mm})\end{array}$ \\
\hline Area I & 2.07 & 2.75 & 7.36 & 14.7 \\
Area II & 2.59 & 2.82 & 7.41 & 12.5 \\
\hline
\end{tabular}

in open areas, so the closer the mean is to the maximum size the more we may expect it to be an area with mostly open terrain.

One may note that the positive mean residual values for both areas in Table 1 imply that model estimates generally underestimate the real crystal size. This gives support to the implicit assumption of the model that for any greyscale value brighter than the baseline value, surface hoar is assumed to grow. The general underestimation of crystal sizes indicates that the model applies the assumption in a relatively conservative manner.

Crossplots for single days which have been spatially estimated using the model may be found in Fig. 7. In Fig. 7a, one can see a distinct widening trend in real-world size variance for the lighter greyscale values. This demonstrates that while trees account for much of the variance in the area, open areas with light greyscale values produce a range of real sizes not able to be predicted by this model. We expected this, as our model only accounts, via greyscale, for the spatial effects of trees.

We then confirmed this relation outside of the January formation cycle. During the surface hoar formation cycle in February 2009, we obtained an average single sample from an average open location and 47 additional points in the area, all using the same sampling method as in January. This new area was at a different location ( $150 \mathrm{~km}$ to the northwest), and a different aspect (south facing) to demonstrate the model holding across differing time, aspect, and location.

The single point contained surface hoar with mean size $5 \mathrm{~mm}$, and that point on Google Earth had a grey 40 value of 0.65 , much whiter than the images from Area I or II. Coincidentally, the area also had the same grey baseline $=0.22$, which we found via a histogram of greyscale values of the image. To obtain this greybaseline, we took the average dark value of the lowest peak of dark grey 40 values in a detailed histogram.

We used the simplified $\Delta$ grey $_{40}$ from Eq. (4). As mentioned above in Sect. 4.6, field methods would also ensure that the dark greybaseline value did, in fact, correspond to $0 \mathrm{~mm}$ so the model can be properly scaled. 

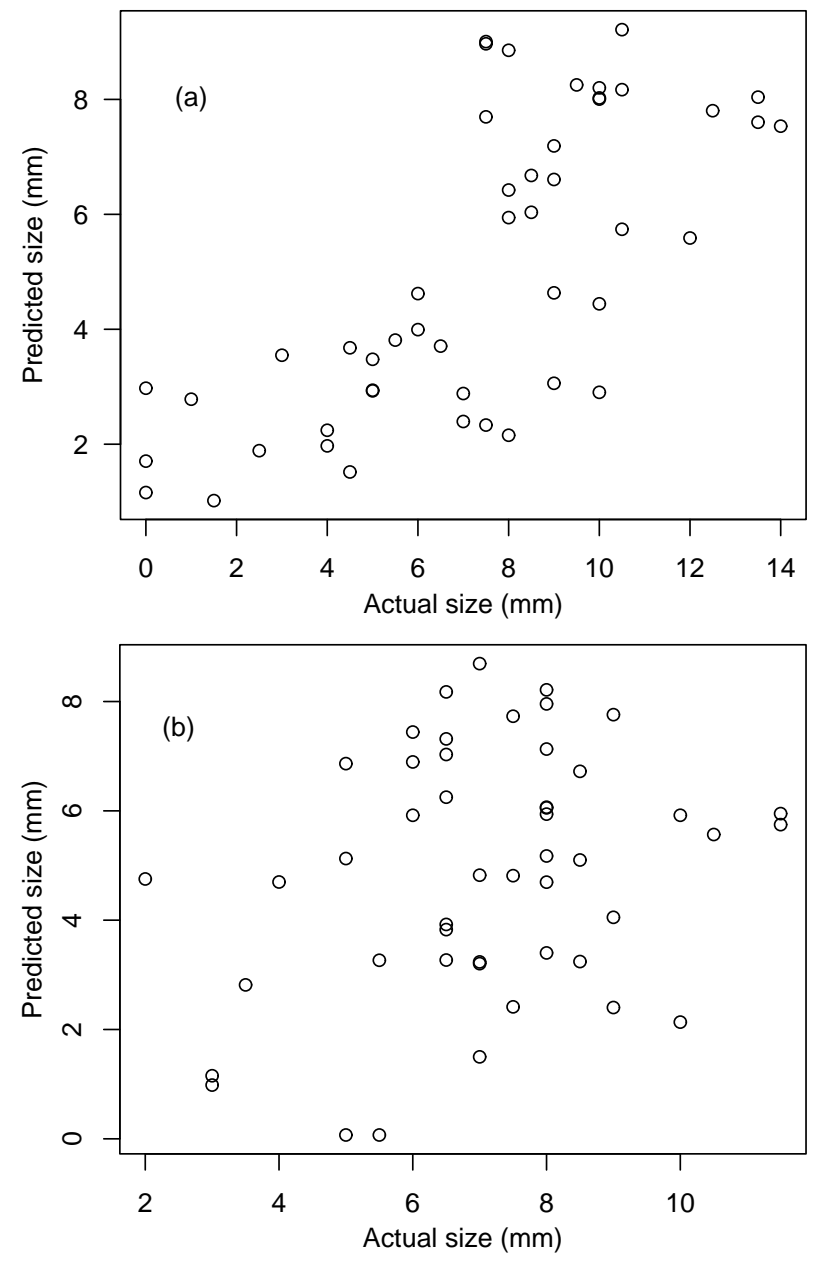

Fig. 7. Crossplots of predicted versus actual surface hoar crystal size for both sample areas. (a) Area I. (b) Area II.

This gives us a grey change per $\mathrm{mm}$ growth $\Delta$ grey $_{40}=0.086 / \mathrm{mm}$. From Eq. (1), we adapted the following model to predict mean sizes for the surrounding area, for this new February layer:

size $_{\text {feb } \_17-20}($ lat, lon $)=\frac{\text { grey }_{40}(\text { lat }, \text { lon })-0.22}{0.086}$

Using Eq. (6), we then predicted the sizes at each of the 47 physical points that we sampled, and we compared the predictions to the actual sampled values. The model predicted the correct size of surface hoar to within $1.5 \mathrm{~mm}$ for $60 \%$ of the points, and to within $2 \mathrm{~mm}$ for $70 \%$ of the points. As the average crystal size from those 47 points also equaled $5 \mathrm{~mm}$, these results are similar to those of the January cycle.

The model produced a mean of absolute value residuals equal to $1.52 \mathrm{~mm}$ - meaning that, as before, actual crystal sizes were generally larger than predicted - and a standard deviation of actual residuals equal to $1.80 \mathrm{~mm}$.
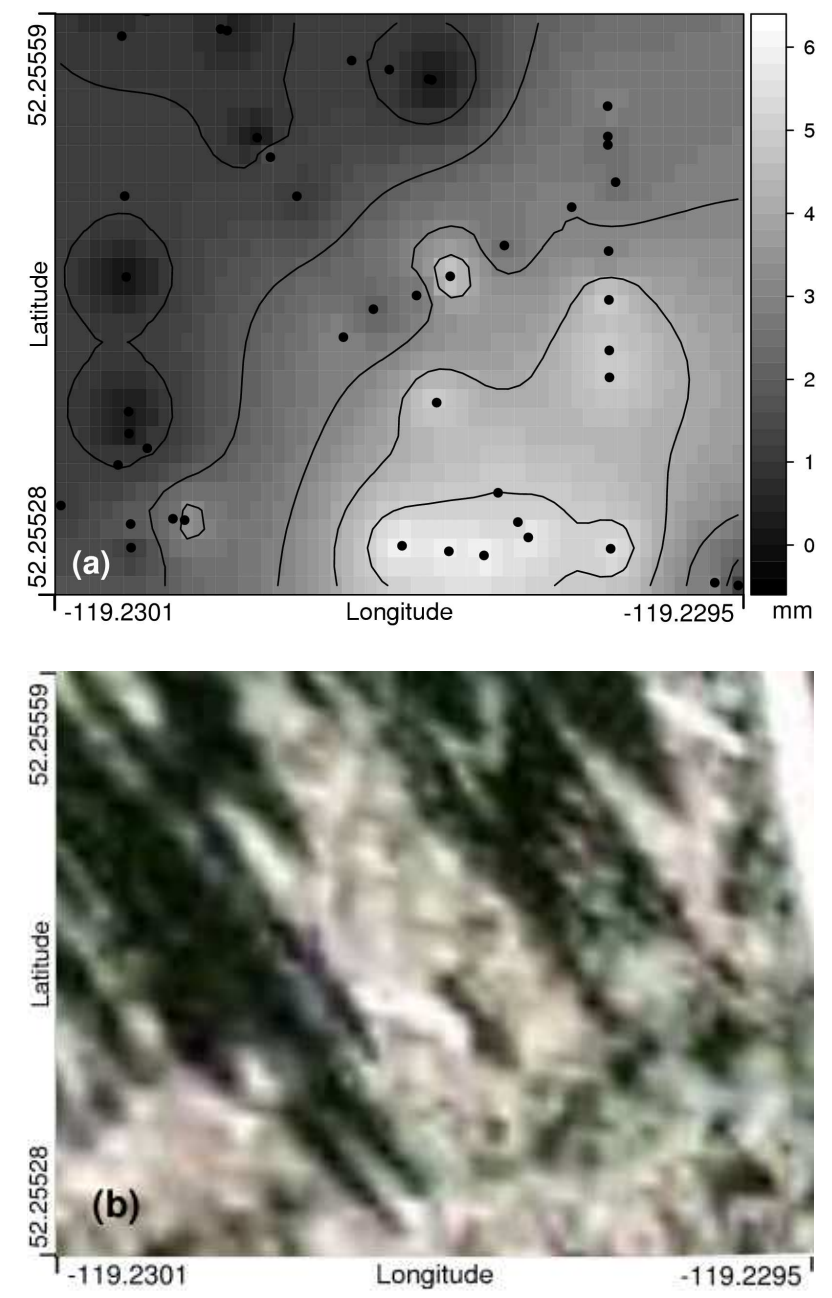

Fig. 8. Area used for verification of the greyscale relation. (a) IDW map of measured mean crystal sizes in the new area sampled on 19 February 2009. Black dots indicate the 47 actual physical measurement and model verification points. Contours have been added for visual clarity. (b) Satellite image corresponding to the sample area used. Note the extreme slant in tree shading projection on this south aspect satellite image. Satellite image is provided under the Google Earth terms of use, copyright 2009 TeleAtlas, 2009 British Columbia, and 2007 Google Earth.

Visually, the IDW map confirms the same shading effect; the satellite greyscale image and corresponding map are shown in Fig. 8.

The similarity in accuracy between the model-building data from January and the predicted February values indicates that the constant increase strategy of using greyscale is extracting as much as can be used. No one variable can account for all of the variance in surface hoar size. But greyscale does rather well, especially considering its ease of use. 


\section{Discussion}

When talking about millimeter-sized changes in surface hoar crystals, separating the amount of change caused by large scale processes versus those associated with small scale variance (which appears as stochastic variance at best when captured at a resolution of one sample per $100 \mathrm{~m}$, for example) can be extraordinarily difficult, if not impossible (Hägeli and McClung, 2000; Campbell et al., 2004; Campbell and Jamieson, 2007).

This highlights the fundamental distinction between measuring a process which affects surface hoar formation, and mapping the result of that process over terrain. The latter, especially, may occur both as a descriptive and as a discovery method.

In this paper, we do not measure a process and then extrapolate it over terrain to create our model; rather, we focus only on mapping and prediction of surface hoar crystal size via satellite photo greyscale values. And we focus on that only for specific instances: sparsely treed, relatively sheltered, and small areas.

Qualitatively, the basis of this model lies upon capturing a radius-of-effect for trees at a single point. Radiation balance, air temperatures, humidity, surface temperatures - the variation of all of these well-known physical quantities is exceptionally difficult to estimate spatially at these small scales. A physically successful method of spatial prediction may use these values in the future. However, we cannot yet quantitatively obtain these values on small spatial scales for noninstrumented areas, much less with the same ease and accuracy with which we can for greyscale.

A result of this study is the demonstration of a possibly useful strategy to find relations with spatial effects over terrain. This strategy, as outlined throughout the paper, has two parts. First, as instrumentation for huge numbers of spatial points can be impractical at best, one may select areas where the environment influences key variables. Sparse forests, though in and of themselves interesting as skiing areas, also represent the control of wind and relative consistency in the major formative process - skyview - from day to day. Second, though weather data may be most desirable for estimation purposes, it remains spatially elusive at small scales, and so using other augmenting sources of data such as greyscale can help estimate a large and useful amount of variance in crystal size.

Here, we demonstrate the accuracy of the model within a $40 \mathrm{~m} \times 40 \mathrm{~m}$ area surrounding the single point sample. Though this produces estimates at the most useful -i.e. skier - scale, larger scaling may be limited. Scaling the model into a significantly different and totally unsampled drainage or aspect would probably not only affect the relative shading in the satellite photos, but also introduce the effects of the more large-scale formation processes which have not been directly accounted for here.
For an illustrative example of these larger scale effects, examine the large grove of trees in the satellite photo for Area I (Fig. 2). The cluster visually provides shade in the satellite photos and appeared as a mechanism of storing and enhancing daytime heat in the left temperature map in Fig. 5. However, the lack of trees in the photo on the slope immediately to the north in Area I does not help predict its convex north facing structure which so effectively allows the slope to continue cooling in daytime. Aspect has already been shown to have an effect on surface hoar size (Cooperstein, 2008), but aspect does not get captured by satellite greyscale values. A bright greyscale area on a South slope may have the same value in our model as a bright greyscale area on a North slope, but the two areas will probably produce different crystal sizes. Sky view, though partially captured by shading in this study, ultimately depends on more factors than greyscale shading can provide.

As the number of trees per area increases, shading increases and this model will indicate small surface hoar growth, if any. As the number of trees per area decreases, the mean size of surface hoar will increase according to the model, and also by the intuitive concept of increased net outgoing longwave radiation.

However, with no trees or nearly no trees the effect of shading will become less applicable and other effects such as aspect (Cooperstein, 2008), moisture supply (Colbeck et al., 2008), and wind (Hachikubo and Akitaya, 1997; Feick et al., 2007) may become the main determining factors of spatial variance in crystal size. As discussed above, the notably larger actual surface hoar sizes in lighter greyscale valued areas - larger than our model could capture, that is - indicate that in areas with no large shading variance, other formative factors dominate.

Furthermore, when longwave-tree interactions are not the dominating factor within forests - say, a cloud covers the sparse forest in the evening, or very high winds push through the trees - entirely different conditions will result. This fact, true for all poorly understood natural processes, indicates that this model should not be used for multi-kilometer scale modelling.

These open area formative factors oftentimes do not even scale within mountain ranges, much less across them (Hägeli and McClung, 2007). Thus, a general physical solution to the spatial prediction problem remains challenging and elusive.

Even non-formative sources of error such as photography resolution, angle of tress relative to the photography, accuracy of the GPS used to obtain the relation between size max $_{\text {m }}$ and the greyscale value, and other factors can all contribute to how well the model works at any given location. 


\section{Summary and outlook}

This work shows the small-scale spatial variability of surface hoar crystal size in sparse forests, and accounts for part of that variability using one factor: sky view. We developed a spatially predictive linear model which estimates surface hoar crystal size using averaged greyscale values in a dry land satellite image. Physically, this mechanism relates the size of surface hoar to its amount of sky view, or the amount of tree shading around that point within a $4 \mathrm{~m}$ radius. The relation held across different days and aspects in a January 2009 formation cycle, and also worked in an entirely different area, aspect, and formation cycle in February 2009. The results of the model generally do well at categorizing larger or smaller crystals, and had reasonable error for prediction using only one independent variable.

No previous work has done a two-dimensional spatial examination with this many surface hoar measurement points, much less developed a spatial model for surface hoar size that works at all, on any scale, in any conditions. As a field, we have yet to even set eyes upon extensively measured spatial variance of surface hoar and understand it qualitatively - this skier-scale study represents only a beginning. So, though the highest goal would be to have a model that works everywhere all the time, less than $30 \%$ average error for a new cycle and area represents a fairly reasonable start.

Independently of predictive uses, our data shows the previously unknown extreme crystal size variance to be found at these $40 \mathrm{~m}$ scales. The greyscale relation shows the radius of effect of trees when they are the dominating factor, and it represents the first focus on the spatial surface hoar formation variable of sky view.

Also, this work demonstrates that surface hoar formation research need not necessarily be limited to single points near accurate weather stations. Other augmenting information may be found and coaxed into helping find trends and produce estimates in areas and at scales where precise weather data at each point would be impossible to obtain.

More generally, this study shows the extreme spatial variance of surface hoar sizes on the skier scale. Such variance within these small $40 \mathrm{~m} \times 40 \mathrm{~m}$ areas clearly shows the limitation of trying to project single point estimates over a very large area. Said another way: we cannot usefully spatially map surface hoar formation for skiers over large scales before being able to do so over small ones. With a general prediction of " $5 \mathrm{~mm}$ mean crystal size" for a $40 \mathrm{~m} \times 40 \mathrm{~m}$ area, crystals will still range from $0 \mathrm{~mm}$ to $7 \mathrm{~mm}$, or larger. For the January cycle, " $8 \mathrm{~mm}$ mean crystal size" could mean a range from $0 \mathrm{~mm}$ to $14 \mathrm{~mm}$, all within a small area. This could correspond to triggering ranging from very likely to very unlikely, within the same area described with a single mean. Measuring and noting small terrain effects, and how they actually fit within the spatial variance of a larger area seems more useful than simply striving for a larger scale mean size prediction. One can see the use in mapping and understanding - even one by one - the spatial factors that create such variance.

Our approach here limits wide applicability. But, the results of such an approach can still provide important uses, as this study does for understanding sky view for skiers in trees. And, such an approach - with a semi-controlled environment and additional augmenting data - maximizes the potential to find a useful relation that may be further built upon later. In the same way that a handful of factors may be identified and measured for one single point of surface hoar growth, finding conditions and areas that allow identification and measurement of only a few spatial factors at once may be key to pushing our physical and single-point surface hoar knowledge into the spatial realm.

Acknowledgements. For financial support, the authors gratefully thank the Natural Sciences and Engineering Research Council of Canada, HeliCat Canada, the Canadian Avalanche Association, Mike Wiegele Helicopter Skiing, Teck Mining Company, Canada West Ski Areas Association, the Association of Canadian Mountain Guides, Backcountry Lodges of British Columbia, and the Canadian Ski Guides Association.

For logistical support we thank the Avalanche Control Section of Glacier National Park and Mike Wiegele Helicopter Skiing. For careful field work, we thank Thomas Exner, Mike Smith, Cameron Ross, Ali Haeri, Mark Kolasinski and Jordan Stiefvater.

Edited by: J. M. Vilaplana

Reviewed by: two anonymous referees

\section{References}

Brown, M., Grimmond, S., and Ratti, C.: Comparison of methodologies for computing sky view factor in urban environments, International Society of Environmental Hydraulics, Internal Report Los Alamos National Laboratory, Los Alamos, NM, LA-UR-014107, 2001.

Campbell, C. and Jamieson, B.: Spatial variability of slab stability and fracture characteristics within avalanche start zones, Cold Reg. Sci. Technol., 47, 134-147, 2007.

Campbell, C., Jamieson, B., and Hägeli, P.: Small-scale mapping of snow stability: If not, why not, Avalanche News, Canadian Avalanche Association, Revelstoke, BC, Canada, 71, 45-49, 2004.

Cheng, C. and Shiu, C.: Frost formation and frost crystal growth on a cold plate in atmospheric air flow, Int. J. Heat Mass Tran., 45, 4289-4303, 2002.

Colbeck, S.: On the micrometeorology of surface hoar growth on snow in mountainous area, Bound.-Lay. Meteorol., 44, 1-12, 1988.

Colbeck, S., Jamieson, B., and Crowe, S.: An attempt to describe the mechanism of surface hoar growth from valley clouds, Cold Reg. Sci. Technol., 54, 83-88, 2008.

Cooperstein, M.: The effects of slope aspect on the formation of surface hoar and diurnally recrystallized near-surface faceted 
crystals, MSc thesis, Department of Earth Sciences, University of Montana, Montana, 2008.

Cressie, N.: Statistics for Spatial Data, Revised Edition, John Wiley and Sons, Inc., 1993.

Deems, J., Fassnacht, S., and Elder, K.: Fractal distribution of snow depth from LiDAR data, J. Hydrometeorol., 7, 285-297, 2006.

Feick, S., Kronholm, K., and Schweizer, J.: Field observations on spatial variability of surface hoar at the basin scale, J. Geophys. Res., 112, F02002, doi:10.1029/2006JF000587, 2007.

Föhn, P.: Simulation of surface-hoar layers for snow-cover models, Ann. Glaciol., 32, 19-26, 2001.

Hachikubo, A.: Numerical modelling of sublimation on snow and comparison with field measurements, Ann. Glaciol., 32, 37-42, 2001.

Hachikubo, A. and Akitaya, E.: Effect of wind on surface hoar growth on snow, J. Geophys. Res., 102, 4367-4373, 1997.

Hägeli, P. and McClung, D.: A new perspective on computer-aided avalanche forecasting: Scale and scale issues, Proceedings of the International Snow Science Workshop, 66-73, 2000.

Hägeli, P. and McClung, D.: Expanding the snow-climate classification with avalanche-relevant information: initial description of avalanche winter regimes for southwestern Canada, J. Glaciol., 53, 266-276, 2007.

Heierli, J., Gumbsch, P., and Zaiser, M.: Anticrack nucleation as triggering mechanism for snow slab avalanches, Science, 321, 240-243, 2008.

Höller, P.: Tentative investigations on surface hoar in mountain forests, Ann. Glaciol., 26, 31-34, 1998.

Jamieson, B. and Geldsetzer, T.: Avalanche Accidents in Canada, Volume 4: 1984-1996, The Canadian Avalanche Association, 1996.

Jamieson, B. and Schweizer, J.: Texture and strength changes of buried surface hoar layers with implications for dry snow-slab avalanche release, J. Glaciol., 46, 151-160, 2000.
Kronholm, K. and Birkeland, K.: Reliability of sampling designs for spatial snow surveys, Comput. Geosci., 33, 1097-1110, 2007.

Lang, R., Leo, B., and Brown, R.: Observations on the growth process and strength characteristics of surface hoar, Proceedings of the International Snow Science Workshop, Aspen, Colorado, 188-195, 1984.

Lang, R. M.: Studies on Surface Hoar: Formation and Physical Properties, Master of Science in Engineering Mechanics, Montana State University, Bozeman, Montana, 1985.

Lehning, M., Bartelt, P., Brown, B., and Fierz, C.: A physical SNOWPACK model for the Swiss avalanche warning, Part III: meteorological forcing, thin layer formation and evaluation, Cold Reg. Sci. Technol., 35, 169-184, 2002.

McClung, D. and Schaerer, P.: The Avalanche Handbook, 3rd edn., The Mountaineers Books, Seattle, WA, 2006.

Morstad, B., Adams, E., and McKittrick, L.: Experimental and analytical study of radiation-recrystallized near-surface facets in snow, Cold Reg. Sci. Technol., 47, 90-101, 2007.

Pebesma, E. J. and Wesseling, C. G.: GSTAT: a program for geostatistical modelling, prediction and simulation, Comput. Geosci., 24, 17-31, 1998.

R Development Core Team: R: A Language and Environment for Statistical Computing, R Foundation for Statistical Computing, Vienna, Austria, available at: http://www.R-project.org, ISBN 3900051-07-0, 2006.

Shea, C. and Jamieson, B.: Star: An efficient snow point-sampling method, Ann. Glaciol., 51(54), 64-72, 2010.

Sicart, J., Pomeroy, J., Essery, R., Hardy, J., Link, T., and Marks, D.: A sensitivity study of daytime net radiation during snowmelt to forest canopy and atmospheric conditions, J. Hydrometeorol., 5, 774-784, 2004. 